\title{
Suppression of Resonant Auger effect with chirped x-ray free-electron laser pulse
}

\author{
Yu-Ping Sun ${ }^{1}$, Quan Miao ${ }^{2}$, Ai-Ping Zhou ${ }^{1}$, Rui-Jin Liu ${ }^{1}$ and Faris Gel'mukhanov ${ }^{3}$ \\ ${ }^{1}$ School of Physics and Optoelectronic Engineering, Shandong University of Technology, 250049 \\ Zibo, China \\ ${ }^{2}$ College of Electronics, Communication and Physics, Shandong University of Science and \\ Technology, 266590 Qingdao, China \\ ${ }^{3}$ Theoretical Chemistry and Biology, School of Biotechnology, Royal Institute of Technology, \\ Roslagstullsbacken 15, S-10691 Stockholm, Sweden \\ E-mail:sunyuping@sdut.edu.cn
}

\begin{abstract}
We study the Auger effect induced by strong x-ray free-electron lasers (XFELs) propagating through the resonant argon vapors through solving the Maxwell-Bloch equations numerically. The simulations are based on the three-level system with the carrier frequency tuned in the $2 p_{3 / 2}-4 s$ resonance. It is shown that the Auger branching is sentive to the pulse area and duration. The relative Auger yield can be suppressed in the course of pulse propagation due to the interplay between the Auger decay and stimulated emission. Further suppression can be achieved by chirping the initial pulse, which is more effective for long-pulse case. In addition, the sign and magnitude of the chirp rate play important roles in pulse reshaping and Auger emission.
\end{abstract}

\section{Introduction}

The invention of x-ray free electron lasers (XFELs) has opened unique opportunities to research nonlinear $\mathrm{x}$-ray science due to its extreme pulse characteristics beyond the limitations of current synchrotron radiation light sources, such as the ultrahigh intensity (up to $10^{18} \mathrm{~W} / \mathrm{cm}^{2}$ ) and ultrashort pulse duration (typically a few femtoseconds)[1-3]. The novel peoperties of x-rays enable us to study the radiation-matter interaction at previously unreachable dimensions using varisou X-ray spectroscopy techniques[4-8]. Auger effect is a common analytical tool of x-ray spectroscopy in surface and material science because it is the major effect determining the dynamics of the atoms and molecules in core-hole states[9]. Recently, the resonant Auger effect induced by intense XFELs has extensively been studied owing to its short duration in the same order as the characteristic lifetime of core-hole states[10-15]. It has demostrated that the Auger spectra can be modified due to the interplay betwwen radiative and nonradiative decay channels[12]. However, the emission of Auger electrons is undesirable for the resonant propagation of XFEL lasers with high intensity, because the Auger ionization followed by the Coulomb explosion is the major reason of radiation damage of the sample[16]. How to suppress the Auger effect has become a key question in the case of resonant x-ray scattering. 
Rohringer and Santra [17] studied how the stimulated emission affects the Auger yield without taking into account the XFELs propagation effects, assuming an optically thin medium. Our previous work shows that the propagation of strong XFEL short-pulse is accompanied by the pulse compression [18-20]. The main reason for this is the intrinsic chirp produced in the course of pulse propagation, which interacts with the dispersion properties of the medium, increasing or decreasing total pulse dispersion and consequently changing the dynamics of the pulse propagation. However, it is more difficult to compress the long-pulse only replying on the intrinsic chirp during propagation[20]. A question arises: how to modify the dynamics of the XFELs and the relaxation processes if we introduce chirp rate in the initial pulse?

The aim of this work is mainly to investigate the role of pulse propagation and initial chirp rate on the resonant Auger effect by numerically solving the coupled Maxwell-Bloch equations. The paper is organized as follows: in Section 2, we present the theoretical model and computatinal details. In Section 3, we analyze the resonant Auger effect induced by unchirped and chirped x-ray free-electron pulses. Finally, we offer some conculsions in Section 4.

\section{Theoretical model}

To exemplify our theory, we select the gas phase Ar as the medium. The ground state of Ar atom has the following electronic configuration: $|0\rangle=(1 s)^{2}(2 s)^{2}(2 p)^{6}(3 s)^{2}(3 p)^{6}$. The carrier frequency of incident pulse is tuned in resonance with the $2 p_{3 / 2}-4 s$ transition $(\hbar \omega=$ $244.3 \mathrm{eV}$ ), which can be matched using hard x-ray at LCLS and SACLA. In this case, the possible electronic transitions induced by strong XFEL pulses are shown schematically in Figure 1. When the x-ray photons with suffcient energy shine Ar atoms, the electrons of inner $2 p$ shell is excited to outer empty $4 \mathrm{~s}$ shell and the electrons of $3 s$ or $3 p$ orbitals can be removed completely from the atom to become photoelectrons. The direct photoionization of $2 p_{3 / 2}$ electrons can be ignored because the photo frequency is below the ionization threshold of the $2 p_{3 / 2}$ electrons. The core-excited state $|1\rangle=\left|2 p_{3 / 2}^{-1} 4 s^{1}\right\rangle$ with inner shell vacancies is not stable, and the electrons from $3 \mathrm{~s}$ or $4 \mathrm{~s}$ orbitals will decay and fill the vacancies, which opens two amplified spontaneous emission channels: $|1\rangle \rightarrow|0\rangle$ and $|1\rangle \rightarrow|2\rangle=\left|3 s^{-1} 4 s^{1}\right\rangle$. The energy available in this deexcitation process can be released through two competitive channels: radiative decay and Auger decay. Our theoretical model is based on strict numerical solutions of the coupled density matrix and Maxwell's equations for three-level atoms: the ground state $|0\rangle$, core-excited state $|1\rangle$, and two final states $|0\rangle$ and $|2\rangle$. The frequency of the pump field is assumed to be in resonance with the $x$-ray absorption transition $|0\rangle \rightarrow|1\rangle$.

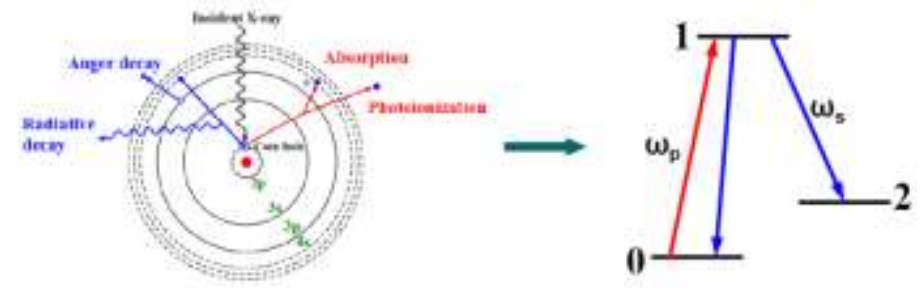

Figure 1. Scheme of electronic transitions (left) and the corresponding three-level model (right)[左图待修改！]

\subsection{Maxwell-Bloch equations for three-level system}


The atomic system is treated quantum mechanically with density matrix equations, and the electromagnetic radiation is described classically by Maxwell equations. For our studied system, the spontaneous decay rate can be ignored in simulations because it is much smaller compared with the Auger relaxation rates. Taking into account the Auger decay and direct photoionization induced by strong XFEL field, populations of the states $\rho_{n n}$ and the coherences $\rho_{m n}(m \neq n)$ obey the following diensity matrix equations

$$
\begin{aligned}
& {\left[\frac{\partial}{\partial t}+\Gamma+\gamma_{p h}^{(1)}(t)\right] \rho_{11}=W=2 \mathfrak{J} \sum_{n=0,2} V_{1 n} \rho_{n 1}} \\
& {\left[\frac{\partial}{\partial t}+\Gamma_{f}+\gamma_{p h}^{(2)}(t)\right] \rho_{22}=2 \Im\left(V_{21} \rho_{12}\right)} \\
& \frac{\partial}{\partial t}\left(\rho_{00}+\rho_{11}+\rho_{22}\right)=-\left[\Gamma+\gamma_{p h}^{(1)}(t)\right] \rho_{11}-\left[\Gamma_{f}+\gamma_{p h}^{(2)}(t)\right] \rho_{22}-\gamma_{p h}^{(0)}(t) \rho_{00} \\
& {\left[\frac{\partial}{\partial t}+\gamma+\frac{1}{2}\left(\gamma_{p h}^{(1)}(t)+\gamma_{p h}^{(0)}(t)\right)\right] \rho_{10}=i V_{10}\left(\rho_{11}-\rho_{00}\right)-i V_{12} \rho_{20}} \\
& {\left[\frac{\partial}{\partial t}+\gamma+\gamma_{f}+\frac{1}{2}\left(\gamma_{p h}^{(1)}(t)+\gamma_{p h}^{(2)}(t)\right)\right] \rho_{12}=i V_{12}\left(\rho_{11}-\rho_{22}\right)-i V_{10} \rho_{02}} \\
& {\left[\frac{\partial}{\partial t}+\gamma_{f}+\frac{1}{2}\left(\gamma_{p h}^{(0)}(t)+\gamma_{p h}^{(2)}(t)\right)\right] \rho_{20}=-i V_{21} \rho_{10}+i \rho_{21} V_{10}}
\end{aligned}
$$

Here, $\omega_{m n}=\left(E_{m}-E_{n}\right) / \hbar$ are the resonant frequencies. $V_{m n}=-\left(E(t, z) d_{m n} / \hbar\right) e^{i \omega_{m n} t}$ is matrix element of the operator of dipole interaction between the atoms and the X-ray field. The density matrix is normalized for unit for $t \leq 0$. It should be noted that the total number of resonant atoms $\rho_{00}+\rho_{11}+\rho_{22}$ decreases for $t>0$ because of the Auger decay and direct ionization. $\Gamma$ and $\Gamma_{f}$ denote the Auger relaxation rates of the core-excited state and final state, respectively. For the Auger decay of the coherences, $\gamma=\Gamma / 2, \quad \gamma_{f}=\Gamma_{f} / 2$. The photoionizaiton rate $\gamma_{p h}^{(k)}(t)=\sigma_{k} s(t)=\sigma_{k} I(t) /(\hbar \omega)$, depends on the photoionizaiton cross section $\left(\sigma_{k}\right)$ of the state $\mathrm{k}$ and the photon flux $(s(t))$. The intensity $I(t)=|E(t) H(t)|$.

The evolution of the XFEL field propagating along the $z$ axis is described through 1D Maxwell's equations, which ignore the transverse inhomogeneity of the pulse.

$$
\frac{\partial E}{\partial z}+\mu_{0} \frac{\partial H}{\partial t}=0, \quad \frac{\partial H}{\partial z}+\varepsilon_{0} \frac{\partial E}{\partial t}=-\frac{\partial P}{\partial t}
$$

Maxwell's equations take into account the incident field as well as all other fields generated in the course of pulse propagation, while the role of backward-travelling wave is negligible [21]. Density matrix equations (1) and Maxwell's equations (2) are coupled with each other through the interaction $V_{m n}$ and the light-induced polarization 


$$
P(t, y)=N \operatorname{Tr}(d \rho)=2 N \Re\left(d_{10} \rho_{01} e^{i \omega_{10} t}+d_{12} \rho_{21} e^{i \omega_{12} t}\right)
$$

where $N$ is the concentration of the atoms.

\subsection{Relative Auger yield}

It should be mentioned that the effect of direct photoionization on the pulse reshaping and the Auger yield can be ignored for our studied case due to the rather long photoionization time in comparison with the pulse duration and the Auger decay time[15, 20]. The nonresonant interaction between XFEL and ionized atoms has also not been included in our simulations. This approximation is valid because the amount of ions is much smaller than the intact atoms for paopagation distances and pulse intensities studied here[15]. Thus, the equations for the population of the core-excited state and for the total population of the intact atoms can be rewritten in a physically clear form as

$$
\begin{aligned}
& \frac{\partial}{\partial t} \rho_{11}=-\Gamma \rho_{11}+W=-\left(\Gamma \rho_{11}+W_{E}\right)+W_{A} \\
& \frac{\partial}{\partial t}\left(\rho_{00}+\rho_{11}+\rho_{22}\right)=-\Gamma \rho_{11}-\Gamma_{f} \rho_{22}
\end{aligned}
$$

The population of the core-excited state $\rho_{11}$ is governed by the Auger decay and so-called field work $W$, which experiences sign-changing modulations for strong XFEL fields. It is instructive to divide $W$ into positive $\left(W_{A}>0\right)$ and negative parts $\left(-W_{E}<0\right)$, which are responsible for the absorption and stimulated emission induced by strong XFEL field, respectively. The core-excited state is populated through the absorption $\left(W_{A}\right)$ and is depopulated due to Auger decay $\left(\Gamma \rho_{11}\right)$ and the stimulated emission $\left(W_{E}\right)$. Considering the work function of the field $W=2 \mathfrak{I} \sum_{n=0,2} V_{1 n} \rho_{n 1}$ depends on the strength of the electromagnetic field through the interaction operator $V_{m n}$, a competition mechanism exists between the stimulated emission and Auger decay channels. The first impression says the Auger branching ratio can be suppressed due to the strong stimulated emission for the strong XFEL fields. This allows us to introduce relative Auger yield $\eta(z)$ to describe the relative strength of the Auger branching.

$$
\eta(z)=\frac{\rho_{\text {aug }}(z)}{\rho_{\text {aug }}(z)+\rho_{E}(z)}, \quad \rho_{\text {aug }}(z)=\Gamma \int_{-\infty}^{+\infty} \rho_{11}(z, t) d t, \quad \rho_{E}(z)=\int_{-\infty}^{+\infty} W_{E}(z, t) d t
$$

Here we focus our attention only on the partial Auger yield caused by the decay of the core-excited state and the smaller Auger yield of the final state is not concerned.

\subsection{Computational details}

The coupled Maxwell-Bloch equations are solved using an iterative predictor-corrector finite-difference time-domain (FDTD) method. In the simulations, we use the experiment 
frequencies $\omega=\omega_{10}=244.3 \mathrm{eV} \quad[22], \quad \omega_{12}=219.28 \mathrm{eV} \quad$ [23]. The time-step of integration $\mathrm{dt}=2 \pi /\left(120 \omega_{0}\right)=1.412 \times 10^{-4} f s$, and the space-step of integration $\mathrm{dz}=2 \cdot c \mathrm{dt}=8.476 \times 10^{-5} \mu \mathrm{m}$. To make the simulations closer to the real system, the transition dipole moments $d_{10}$ and $d_{21}$ are obtained based on the experimental cross section [24, 25] and ab initio simulations [26]. $d_{10}=0.137 a . u$.[19], $d_{21}=0.322 a . u$. [19]. The Auger broadening of the core-excited state and final state $\hbar \Gamma=0.12 \mathrm{eV}[22,27], \hbar \Gamma_{f}=0.076 \mathrm{eV}[28]$.

In order to investigate the role of chirped pulse on the pulse reshaping and Auger yield, we introduce linear chirp rate in the initial field with a hyperbolic-secant functional envelop

$$
E_{x}(z=0, t)=E_{0} \operatorname{sech}\left[1.76\left(t-z / c-t_{0}\right) / \tau\right] \times \cos \left[\omega t+\frac{1}{2} \chi t^{2}\right]
$$

where the initial phase is assumed as zero. $E_{0}$ is the peak amplitude of the input electric field. $\tau$ is the full width at half maximum (FWHM) of the pulse intensity envelope. The pulse area is directly proportional to the amplitude of the field $E_{0}$ and the pulse duration $\tau$, $\theta=E_{0} \tau d_{10} \sqrt{\pi / 2 \ln 2} / \hbar . \quad \chi=\Delta \omega / \tau$ is the chirp rate. The choice of $t_{0}$ ensures that the pulse penetrates negligibly into the medium at $t=0$. The atoms are assumed to be at rest in its ground state before the light is turned on, i.e., $\rho_{00}=1, \rho_{11}=\rho_{22}=0$ and $\rho_{m n}(m \neq n)=0$.

In order to avoid the large propagation distances and reduce the computational costs, the simulations are performed for a high concentration of Ar atoms $N=2.23 \times 10^{21} \mathrm{~cm}^{-3}$. It is easier to get results for lower concentrations $N^{\prime}$ using the scaling of the propagation distance $y^{\prime}=y N / N^{\prime}[29]$.

\section{Numerical results and disscussion}

\subsection{The suppression of Auger effect duripng chirp-free pulse propagation}

First of all, we investigate the coherent control of the Auger emission during chirp-free pulse propagation. The influence of pulse area on the relative Auger yield is displayed in Figure 2. The pulse duration is set as $2 f_{s}$, which is shorter than the lifetime of the core-excited state $\left(\sim 5.5 f_{s}\right)$. One can see that the evolution of Auger yield during propagation is distrinct for pulse areas of odd multiple and even multiple of $\pi$. When the pulse the pulse duration is equal to odd multiple of $\pi$, the relative Auger yield starts from a relative higher point and exhibits a decreasing trend in the course of pulse propagation. In contrast, for the pulses with a initial area of even multiple of $\pi$, the increase of the Auger branching at the beginning is followed by its decrease during pulse propagation. For long propagation distances, the relative Auger yield changes smoothly and keeps 
a rather lower value for all pulses.
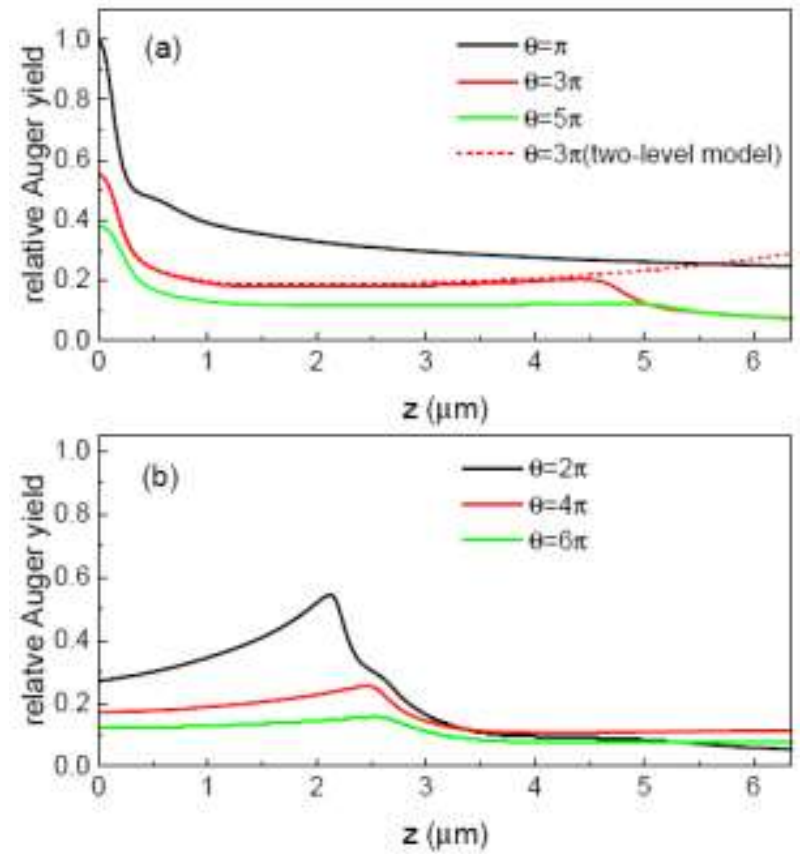

Figure 2. The relative Auger yield versus propagation distance for different pulse area. $\tau=2 f_{s}$.

First, let us pay our attention to the relative Auger yield at the beginning of pulse propagation, where the Auger branching ratio for $\theta=\pi, 3 \pi, 5 \pi$ is higher than that for $\theta=2 \pi, 4 \pi, 6 \pi$. To get a deep insight of physics, it is instructive to look at the so-called field work and the population of core-excited states for $z=0.001 \mu \mathrm{m}$. (seen in Figure 3). In this region, the Stokes field gained through the amplified spontaneous emission channel $|1\rangle \rightarrow|2\rangle$ is negligible, and the final state $|2\rangle$ does not play any role in the work function of the field. The field work exhibits the sign-changing modulations when the field is strong, which is similar as the Rabi oscillations for two-level model[30]. The only difference is that the behavior of the field work shows assymmetric characteristics due to the Auger decay for three-level system. It should be noted that the nodes of the field work (the position of dashed lines in Figure 3(d)) depend sharply on the pulse area. When the pulse area is odd multiple of $\pi$, the absorption is in a dominant position which resulting in a larger population of the core-excited state and hence the higher relative Auger yield. In particular, the Auger branching ratio begin to decrease from $\eta=1$ for $\pi$ pulse (Figure 2 (a)), because the core-excited state is populated through absorption and no stimulated emission occurs for the beginning of pulse propagation (Figure 3). When the pulse duration is equal to even multiple of $\pi$, the Auger branching starts from a relative lower points (Figure 2 (b)) due to the suppression of the population of the core-excited state caused by the oscillating behavior of the field work (Figure 3 ). 
During pulse propagation the evolution of the Auger branching is governed by the competition between Auger decay and stimulated emission.

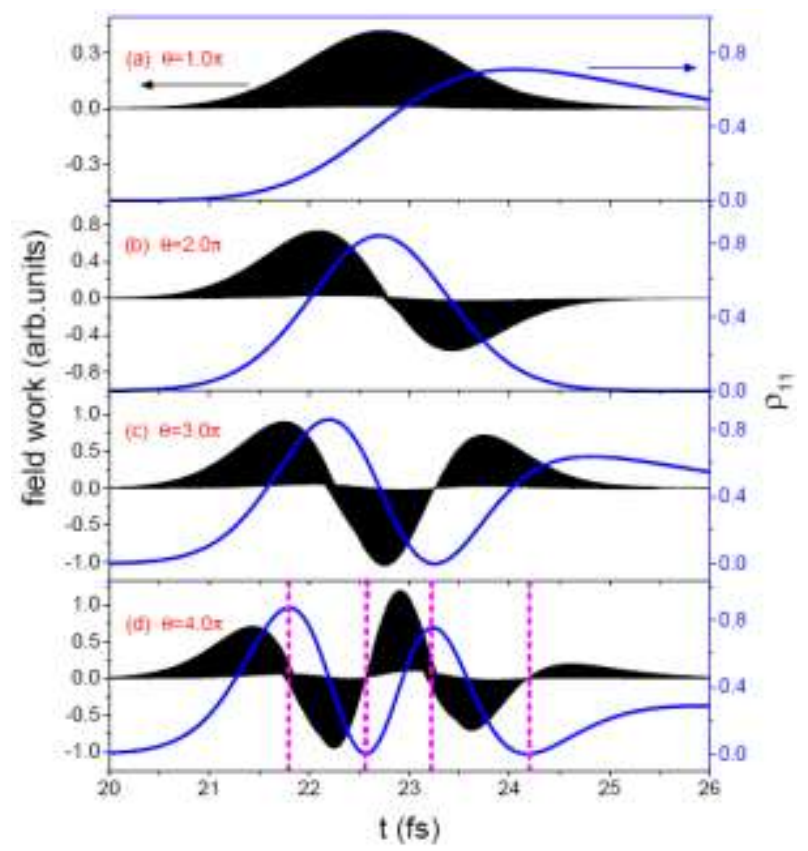

Figure 3. The field work and the population of the core-excited state for the beginning of pulse propagation.

$$
\tau=2 f_{s}, \quad z=0.0001 \mu m, \quad \chi=0 .
$$

As mentioned above, the Auger branching can be strongly suppressed for long propagation distances for all XFEL pulses. To explore the underlying suppression mechanism, we performed the simulations for the two-level model $\left(d_{21}=0\right)$ for $\theta=3 \pi$. Our previous works[ref] show $3 \pi$ XFEL pulse can be strongly compressed during propagation in resonant atomic medium due to the intrinsic chirp[ref]. One can see from Figure 2 (a) that for short distance region, the evolution of relative Auger yield is almost the same for two-level and three-level systems. However, the Auger branching ratio drops down sharply near the critical distance $z_{c}$ for the three-level system, while the two-level approximation is completely invalid for long propagation distances. The physical reason for this can be acquired from the dynamics of the population of the core-excited state. 


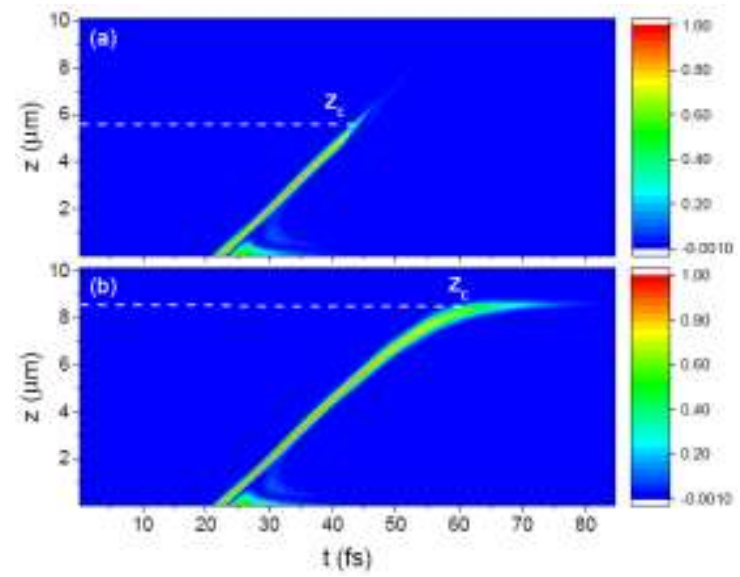

Figure 4. The 2-D map of the population of the core-excited state for (a) three-level and (b) two-level models.

$$
\tau=2 f s, \theta=3 \pi, \chi=0
$$

Figure 4 presents the two-dimensional maps of population of core-excited state for three-level and two-level systems. It is clear that before the certain critical distance, the medium is inverted through the absorption followed by stimulated emission for two schemes. The competition between the Auger decay and stimulated emission channel is responsible for the evolution of the Auger branching in the region of population inversion, where the contribution from the Stokes field is rather weak. In contrast, the Stokes field is gained through amplified spontaneous emission starting from a self-seeding mode for three-level system. Afterwards the destructive interference between the pump field and comparable Stokes field eliminates the population inversion near the critical distance, where the Stokes components are continually enhanced through lasing without inversion. Consequently, for three-level system the auger branching declines sharply near the critical point caused by the abrupt quenching of the population inversion, while no charp changing occurs for Auger yield in two-level atoms due to the absence of Stokes radiation.

To illustrate the dependence of the resonant Auger effect on the pulse duration, the evolution of the relative Auger yield for $3 \pi$ pulses with different width is collected in Figure 5. One can clearly see that the suppression of Auger effect becomes weaker for longer pulse. The reasons for this are twofold. One reason is the amplitude of field is inversely proportional to the pulse duration for a pulse with certain area. For longer pulse, the stimulated emission is suppressed due to the relative lower field intensity. The other and more important reason is due to the competition between Auger decay and stimulated emission. When the pulse duration is shorter than the 
lifetime of the core-excited state, the Auger decay has no enough time to occur compared with relative stronger stimulated emission induced by high intensities. Contrarily, for longer pulses with relative lower intensities, the Auger emission is dominated for the depopulation of the core-excited state.

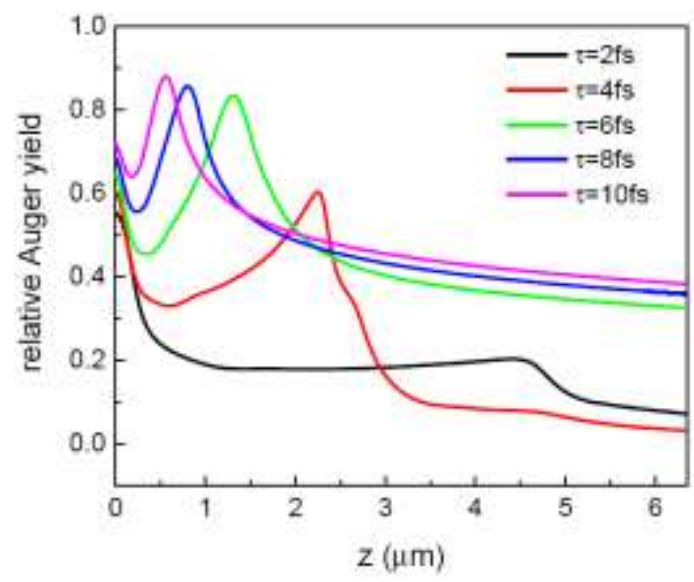

Figure 5. The relative Auger yield versus propagation distance for different pulse duration. $\theta=3 \pi, \chi=0$.

Let us give a short summary of the dependent relationship of the resonant Auger effect with the dynamical parameters of strong XFEL pulses. At the beginning of pulse propagation, the Auger yield is sensitive to the pulse area. During propagation, the relative Auger yield is dominated by the competition between stimulated emission and Auger decay. In the amplified spontaneous emission region, the relative Auger yield is suppressed due to stimulated emission, while in the lasing without inversion region, the relative Auger yield is suppressed due to the population quenching of the core-excited state caused by the interference effect between pump and Stokes components. The compression of Auger branching can be modulated by tuning the pulse duration. The strongest compression of relative Auger yield occurs when the pulse duration is shorter than the lifetime of the core-excited state.

\subsection{The suppression of Auger effect using chirped XFEL pulses}

In this subsection, the goal is to investigate the impact of initial chirp on the Auger branching during pulse propagation. Our previous work shows that the propagation of strong $3 \pi$ XFEL pulse is accompanied by the pulse compression when its duration is shorter than or comparable with the lifetime of the core-excited state. The main reason for this is the intrinsic chirp produced in the course of pulse propagation, which interacts with the dispersion properties of the medium, increasing or decreasing total pulse dispersion and consequently changing the dynamics of the 
pulse propagation. A question arises: is it possible to suppress the Auger emission by introducing chirp in the initial input pulse? It is known that chirping a pulse can lead to a shift of the carrier frequency and a reduction of the peak intensity. The relative Auger yields induced by different chirped pulses are collected in Figure 6.
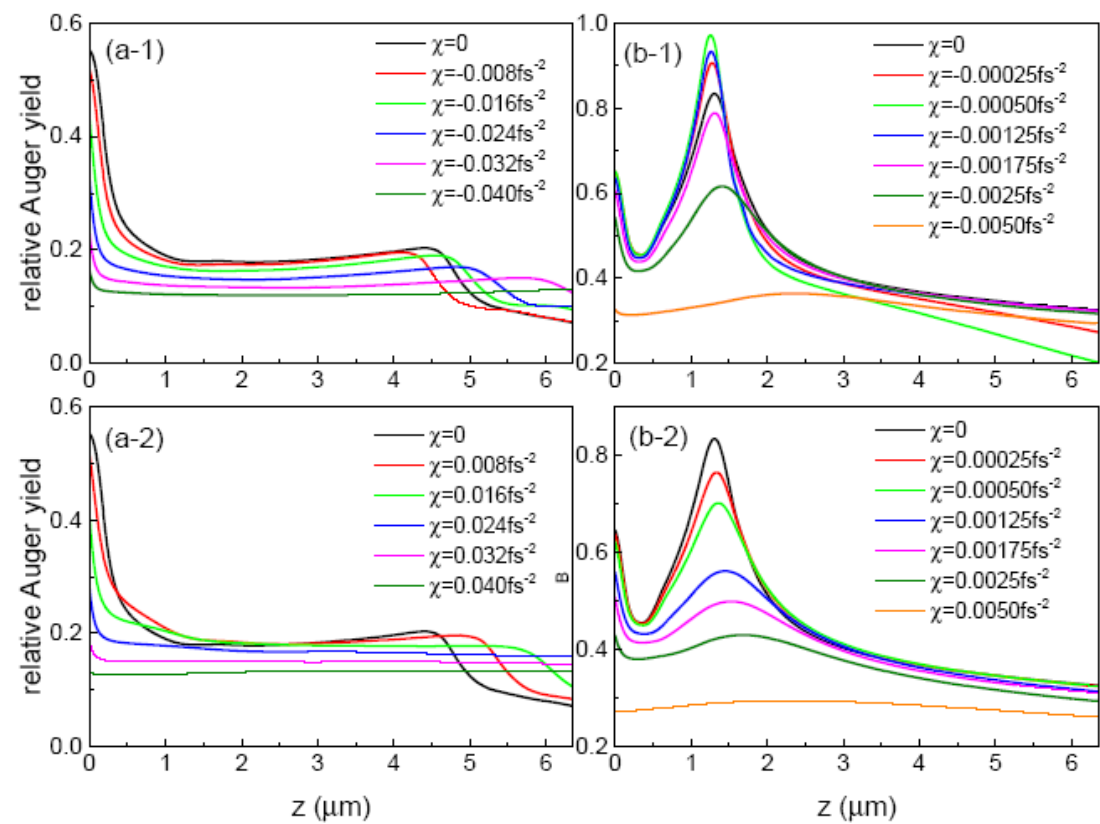

Figure 6. The relative Auger yield versus propagation distance for different pulse duration. $\theta=3 \pi, \chi=0$.

It is shown that the Auger branching can be suppressed through chirping the initial pulses. For short pulses (left panels in Figure 6), the Auger emission is weakened with the increasing the magnitude of the initial chirp rate before the critical distance for both up-chirp and down-chirp cases. As a result of the modulation of the frequency of the field, the gain of the Stokes field decelerates which resulting in a larger critical distance and a relative higher Auger yield for long propagation distance.

As mentioned above, when the pulse duration is beyond of the lifetime of the Auger decay, the Auger branching will be in a dominant position in the decay channels. One can see from Figure 6 that the strong suppression of Auger branching can be achieved by introducing initial chirp for long-pulse case. Moreover, the Auger yield is more sensitive to the sign and magnitude of the chirp-rate for long pulses. To get the implicit physics, let us pay attention to the reshaping of the chirped XFEL pulses (Figure 7). Our previous works[20] show that the XFEL pulse is hard to be compressed only relying on the intrinsic chirp when its width is broader than the lifetime of the core-hole state, which is undesirable for the application and development of XFELs. It is 
clearly seen from Figure 7 that the XFEL pulse can be compressed by chirping the initial pulse compared with chirp free case, while for chirp-free case the intensity of field drops down rapidly due to the dispersion of the medium and the strong Auger decay within the long pulse duration.

The physical reason for this is as follows: the dispersion of the medium and the intrinsic chirp produced during pulse propagation are two competitive mechanisms for the reshaping of the field. An initial chirped pulse can also bring an intrinsic chirp in the course of propagation, which pulls the carrier frequency toward the resonant frequency[31] and hence compensates the dispersion of the medium. As a result, the relative higher intensity and narrower pulsewidth induces dominated stimulated emission and simultaneously weakens the Auger decay probability. It should be noted that for small chirp rate the Auger branching ratio is sensitive to the chirp sign due to the interplay between the initial chirp and the intrinsic chirp induced during pulse propagation.
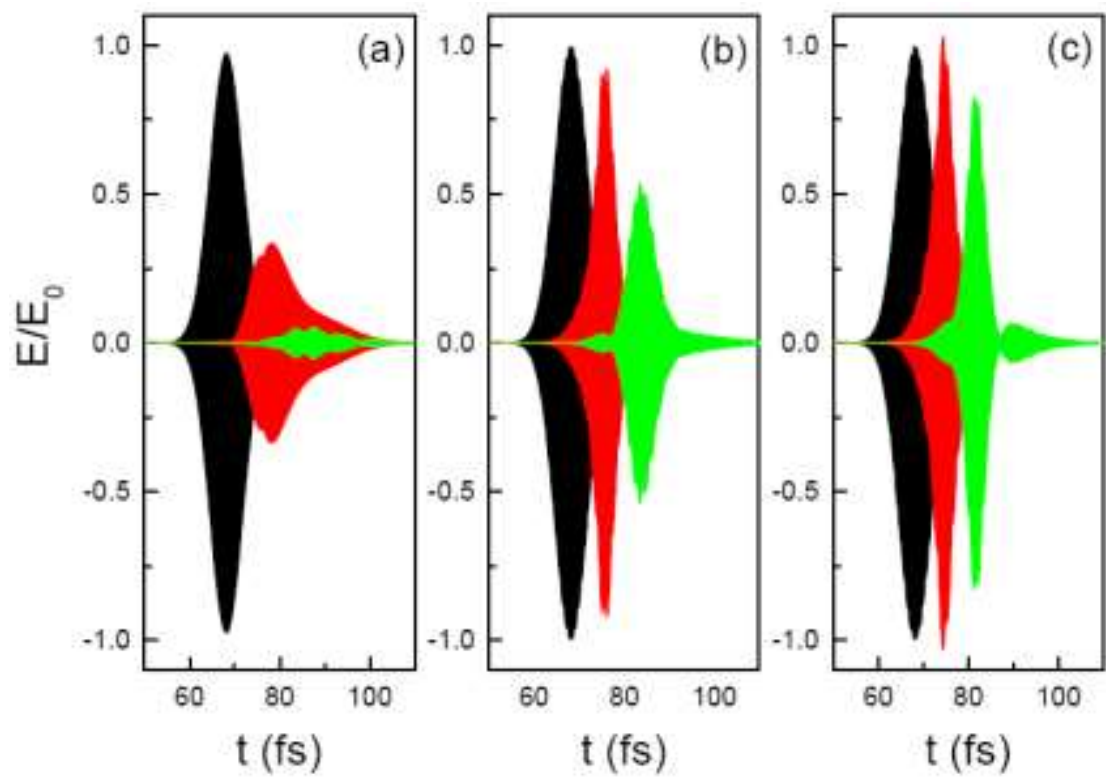

Figure 7. The temporal shapes of the pulse for different propagation lengths $z=0,1.27,2.54 \mu m$ are marked by black, red an green, respectively. $\theta=3 \pi$. (a) $\chi=0$; (b) $\chi=-0.0025 f s^{-2}$; (c) $\chi=0.0025 f s^{-2}$.

\section{Conclusions}

We have studied the resonant Auger effect in the present of strong XFELs propagating through the resonant atomic vapors. As an illustration we performed the simulations for Argon gas based on a three-level mode with the carrier frequency in the $2 p_{3 / 2}-4 s$ resonance. The relative Auger yield is governed by the interplay between two decay channels - Auger decay and stimulated emission. Simulations show that the Auger branching ratio can be suppressed during XFELs propagation. 
Two different suppression mechanisms of Auger decay exist for short and long propagation distances respectively. For short distances, the stimulated emission is the main reason for the suppression of the Auger branching ratio, while for long distances the Auger decay decreases due to the population quenching of the core-hole state caused by the destructive interference between the pump and Stokes field. It is founded that the Auger branching ratio is sensitive to the pulse area, and the evolution is distinct for pulse areas of odd multiple and even multiple of $\pi$. The effective suppression of the Auger yield during pulse propagation occurs only when the pulse duration is shorter than or comparable with the lifetime of the core-hole state. Further suppression of the resonant Auger effect can be achieved by introducing chirp in the initial pulse, especially for long-pulse case. Moreover, the sign and magnitude of the chirp rate play important roles in the pulse compression and Auger decay.

\section{Acknowledgments}

This work was supported by the National Natural Science Foundation of China (grant no. 11204163 and no. 11604181) and Doctoral Foundation of Shandong Province (grant no. BS2013SF018).

\section{References}

[1] C. Bressler and M. Chergui, Chem. Rev. 104, 1781 (2004).

[2] T. Tschentcher, Chem. Phys. 299, 271 (2004).

[3] P. Emma et al., Nat. Photon. 4, 641 (2010).

[4] M. Hoener et al., Phys. Rev. Lett. 104, 253002 (2010).

[5] I. A. Vartanyants et al.,Phys. Rev. Lett. 107, 144801 (2011).

[6] S. Boutet et al.,Science 337, 362 (2012).

[7] D. Nandi et al., Nature 481, 488 (2012).

[8] Ji-Cai Liu et al., J. Phys. B: At. Mol. Opt. Phys. 49, 075602 (2016).

[9] F. Gel'mukhanov and H. Agren, Phys. Rep. 312, 87 (1999).

[10] L. Fang et al.,Phys. Rev. Lett. 105, 083005 (2010).

[11] James P. Cryan et al.,Phys. Rev. Lett. 105, 083004 (2010).

[12] M. N. Piancastelli et al. , Phys. Rev. A 95, 061402 (2017).

[13] S.-B. Zhang, V. Kimberg, and N. Rohringer, Phys. Rev. A 94, 063413 (2016)

[14] R. Sullivan et al., Phys. Rev. A 94, 043421 (2016).

[15] J.-C. Liu et al., Phys. Rev. A 81, 043412 (2010)

[16]S. P. Hau-Riege, R. A. London, and A. Szoke, Phys. Rev. E 69, 051906 (2004).

[17] N. Rohringer and R. Santra, Phys. Rev. A 77, 053404 (2008). 
[18] Y.-P. Sun, J.-C. Liu, and F. Gel'mukhanov, Europhys. Lett. 87, 64002 (2009).

[19] Y.-P. Sun, J.-C. Liu, and F. Gel'mukhanov, J. Phys. B: At. Mol. Opt. Phys. 42, 201001 (2009).

[20] Y.-P. Sun et al., Phys. Rev. A 81, 013812 (2010).

[21] V. Kimberg et al., Phys. Rev. A 74, 033814 (2006).

[22] A. Nilssonet al., ESCA Applied to Free Molecules (North-Holland, Amsterdam, 1969).

[24] R. Haensel et al., J. Phys. (Paris) 32, C4-236 (1971).

[25] M. Nakamura et al., Phys. Rev. Lett. 21, 1303 (1968).

[26] Dalton, References in http://www.kjemi.uio.no/software/dalton/.

[27] D. A. Shaw et al., J. Phys. B 15, 1785 (1982).

[28] S. L. Sorensen et al., Phys. Rev. A 50, 1218 (1994).

[29] S. L. McCall and E. L. Hahn, Phys. Rev. 183, 457 (1969).

[30] Y. Kaluzny et al., Phys. Rev. Lett. 51, 1175 (1983)

[31] X.-H. Song et al., Optics Communications 236, 151 (2004). 